\title{
THE TECTONO-MAGMATIC EVOLUTION OF THE OCCIDENTAL TERRANE AND THE PARAÍBA DO SUL KLIPPE WITHIN THE NEOPROTEROZOIC RIBEIRA OROGENIC BELT, SOUTHEASTERN BRAZIL
}

\section{CLAUDIA SAYÃO VALLADARES, BEATRIZ PASCHOAL DUARTE, MONICA HEILBRON AND DIANA RAGATKY}

\begin{abstract}
The Occidental Terrane is envisaged as the eastern/southeastern reworked margin of the São Francisco/Rio de La Plata plate associated with an E-trending subduction under the Congo plate. The Paraíba do Sul Klippe is part of the Oriental Terrane, envisaged as a portion of the Congo plate. A collisional-stage resulted in intense westward deformation of the Occidental Terrane under intermediate pressure metamorphism (syn-D1+D2 events). A late-collisional stage resulted in subvertical folding and steep shear zones (D3 event). Both stages were associated with voluminous crustal-derived granites. U-Pb and $\mathrm{Sm}-\mathrm{Nd}$ geochronology as well as geochemical and structural data point to three magmatic episodes: 1) a syn-collisional stage $1 ; 2$ ) a syn-collisional stage 2 ; and 3) a late-collisional stage. This paper presents a magmatic evolutionary model for this crustal segment of the Ribeira orogenic belt based on new geological data of Brasiliano granites and data available in the literature.
\end{abstract}

Keywords: Ribeira belt, Brazilian granitoids, geochemistry, Sm-Nd isotopic data

INTRODUCTION The crustal structure of the Ribeira orogenic belt can be defined by two major tectono-stratigraphic terranes, as such: a) the Occidental Terrane is interpreted as the reworked margin of the São Francisco Plate and comprises an autochthonous domain, the so-called Andrelândia thrust system (which records Brasília beltrelated deformation previous events), and the Juiz de Fora thrust system; b) the Oriental terrane is envisaged as part of the Congo Plate and encompasses the Paraíba do Sul Klippe as well as the Costeiro and Cabo Frio tectonic domains.

This paper presents new field, petrographic, geochemical and geochronological (Sm-Nd) data for six Brasiliano-related granitic plutons within the Occidental terrane and the Paraíba do Sul Klippe (Heilbron et al. 1998). These new data, together with published mineral U-Pb geochronology (Machado et al. 1996), field and geochemical data (Junho et al. 1999, Almeida, 1995) support the presently proposed model of three stages (syn-collisional 1, syn-collisional 2 and late collisional), associated with the collision of the São Francisco-Rio de la Plata and Congo plates (Heilbron et al. 1999) during the Brasiliano Orogeny (700-450Ma).

RESULTS The Neoproterozoic magmatism in the Occidental terrane and Paraíba do Sul Klippe (Fig. 1) within the Ribeira belt is of calc-alkaline nature (Fig. 2A) and is mostly represented by granites and subordinated granodiorites (Fig. 2B). Three collisional-related magmatic episodes can be recognized: 1) syn-collisional stage 1 (595$565 \mathrm{Ma})$; 2) syn-collisional stage 2 (565-540 Ma); and 3) latecollisional stage (540-520 Ma).

Syn-collisional 1: (Syn D1+D2 plutons) This stage is represented by abundant peraluminous S-type and metaluminous Itype, strongly foliated and mylonitic granites which occur as NE-SW elongated intrusions within the Juiz de Fora thrust system and Paraíba do Sul Klippe. These features indicate that they were affected by the main deformation phase (D1+D2). The S-type plutons display characteristically abundant supracrustal xenoliths and transitional contacts with the metasedimentary country rocks. These features are suggestive of an anatectic origin. The largest S-type batholith is the syn-D2 Rio Turvo Granite located northwest of Volta Redonda and Barra Mansa (RJ). No geochemical data were yet published for the Rio Turvo Granite. A U-Pb concordant monazite age of $579 \pm 6 \mathrm{Ma}(\mathrm{Ma}-$ chado et al. 1996.) is interpreted as the age of emplacement of the pluton, whereas a discordant titanite ${ }^{207} \mathrm{~Pb} /{ }^{206} \mathrm{~Pb}$ age of $551 \mathrm{Ma}$ suggests that titanite crystallization took place during a later thermal pulse. The peak of metamorphism M1occurred at c. 579 Ma and can be associated with the main $\mathrm{D} 1+\mathrm{D} 2$ deformation event which in turn is taken as the period of main tectonic activity at the central segment of Ribeira orogenic belt. Preliminary Sm-Nd data for the Rio Turvo Granite (Table 1) indicate that some degree of fractionation might have occurred, as pointed out by the low ${ }^{147} \mathrm{Sm} /{ }^{144} \mathrm{Nd}$ values (about 0.07 ) a common value in garnet- and monazite-bearing S-type granites. As a result the $\mathrm{T}_{\mathrm{DM}}$ obtained for this granite may be unrealistic. Alternatively, they might have been generated by melting of mixed sources such as Paleoproterozoic metasediments and 1,0-0,9 Ga basic rocks or still another unknown Mesoproterozoic source.

The Matias Barbosa Granite is the largest I-type pluton in the area occurring south of Juiz de Fora. Contacts between this pluton and the country rocks are mainly tectonic. Biotite is the main mafic phase although hornblende clots occur locally. The granite is generally granoblastic but K-feldspar megacrysts indicate a primary porphyritic nature. Whole-rock geochemistry data (Table 2) show variable composition, ranging from quartz-monzodiorite, granodiorite to granite (Fig. 2B) of predominantly metaluminous nature (Fig. 2C). The REE patterns are strongly fractionated with small Eu anomalies (Fig. $3 \mathrm{~A})$.

Syn-collisional 2: (late-D2 plutons) These are isotropic to slightly foliated I and S-type granites, which outcrop within the Juiz de Fora thrust system. The Pedra Selada, Serra do Lagarto (Junho et al. 1999), and Taquaral (Valladares 1996) I-type plutons are porphyritic to augen granites, whereas the $\mathrm{S}$-type plutons are represented by the Salvaterra Charnoenderbite (Duarte 1998) and Capivara (Almeida 1995) granites, both mainly granular to granoblastic and locally porphyritic.

I-type granites are mainly (hornblende)-biotite porphyritic granites with microcline megacrysts, being homogeneous in composition (granites s.s; Fig. 2B). They often have mafic enclaves and lenses and consist of large NE-SW lenticular bodies. The Pedra Selada and Serra do Lagarto plutons are found nearby Visconde de Mauá (RJ), Bocaina de Minas and Passa Vinte (MG) whereas Taquaral pluton occurs southeast Resende (RJ). The porphyritic fabric of the Taquaral pluton is interpreted as resulting from magmatic syn-deformational flow of a partially crystallized magma. Titanite of the Taquaral Granite (Machado et al. 1996) yields a minimum U-Pb age of $553 \mathrm{Ma}(4 \%$ discordant). No geochemical data were yet published for the Taquaral Granite. Geochemical data for the Pedra Selada and Serra do Lagarto plutons (Junho et al. 1999) point to metaluminous to slightly peraluminous magmas (Fig. 2C) with a strongly fractionated REE pattern and negative Eu anomaly (Fig. 3B).

The S-type granites are isotropic to slightly foliated and show granoblastic to porphyroblastic textures. The Salvaterra

Table 1 - Sm-Nd data for the Rio Turvo and Getulândia granites.

\begin{tabular}{|c|c|c|c|c|c|c|c|}
\hline Sample & $\begin{array}{r}\begin{array}{r}\mathrm{Sm} \\
(\mathrm{ppm})\end{array} \\
\end{array}$ & $\begin{array}{c}\mathrm{Nd} \\
(\mathrm{ppm})\end{array}$ & ${ }^{147} \mathrm{Sm}^{1 / 4} \mathrm{Nd}$ & $\begin{array}{c}]^{1+43} \mathrm{Nd} / /^{1 / 4} \mathrm{Nd} \\
( \pm 1 \sigma)\end{array}$ & $f_{\text {smiNd }}$ & $\begin{array}{l}\mathrm{T} \text { TM } \\
\text { (Ga) }\end{array}$ & Evd (t) ${ }^{2}$ \\
\hline 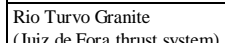 & & & & & & & \\
\hline $\begin{array}{l}\text { sample } 1 \\
\text { senter }\end{array}$ & 18.48 & 143.43 & & $0.511599 \pm 09$ & -0.60 & 1.61 & -11.50 \\
\hline $\begin{array}{l}\text { sample } 2 \\
\text { sample }\end{array}$ & 13.18 & 110.74 & 0.0719 & $0.511584 \pm 16$ & -0.63 & 1.56 & -11.51 \\
\hline $\begin{array}{l}\text { Getulândia Granite } \\
\text { (Paraíba do Sul Klippe) }\end{array}$ & & & & & & & \\
\hline sample 1 & 21.28 & 119.82 & 0.1079 & $0.511722 \pm 11$ & -0.45 & 1.97 & -11.89 \\
\hline
\end{tabular}
Total $(1 \sigma)$ $\mathrm{Nd}$ isotopic compositions were normalized to ${ }^{146} \mathrm{Nd} /{ }^{14} \mathrm{Nd}=0.7219$. For location of the plutons see Figure 1 . $\mathrm{t}=$ crystallisation age.

Analytical procedures: The Sm/Nd isotopic analyses were carried out at the Laboratory of Geochronology of the

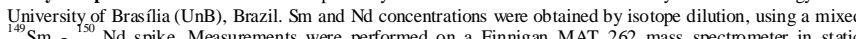
Sm - ${ }^{150} \mathrm{Nd}$ spike. Measurements were performed on a Finnigan MAT 262 mass spectrometer in static multicollection mode. Samples were loaded as phosphates on double Re filament. 

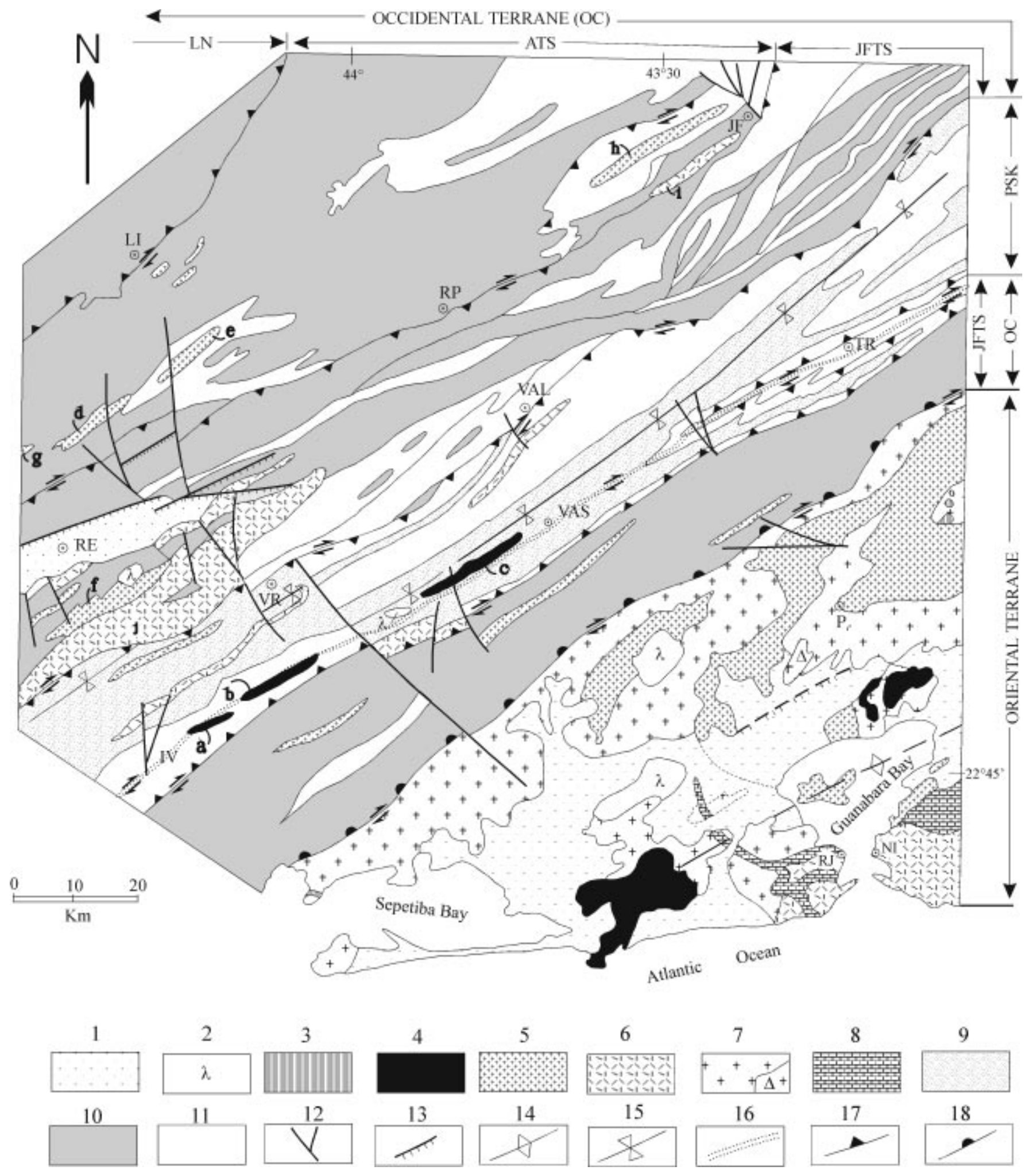

Figure 1 - Geological map of the central segment of the Ribeira belt (modified from Heilbron 1995; Heilbron et al. 1999) 1-Cenozoic cover; 2- MesozoicCenozoic alkaline rocks; Brasiliano granites: 3-Slab detachment-related granites; 4- Late-collisional granites: a-Getulândia Granite, b- Fortaleza Granite, $c$ - Serra do Ipiranga Granite; 5- Syn-collisional granites- late-D2 I-type plutons: $d$-Pedra Selada pluton, e-Serra do Lagarto pluton, $f$-Taquaral Granite; $S$ type plutons: g- Capivara Granite, $h$ - Salvaterra Charnoenderbite; 6- Syn-collisional (syn D1+D2 I-type plutons): $i$ - Matias Barbosa pluton; S-type plutons: $j$ - Rio Turvo batholith; 7 - Magmatic arc rocks including alkaline rocks (D). Basin successions: - 8-Italva; 9- Paraíba do Sul and 10- Andrelândia. 11-pre-1.8 Ga basement associations. Tertiary faults: 12- transfer faults and 13- normal faults; 14- Rio de Janeiro mega-antiformal; 15- Paraíba do Sul megasynformal; 16- Paraíba do Sul shear zone; 17-Major thrusts; 18-Central tectonic boundary. Towns: JF-Juiz de Fora, LI-Liberdade, RP-Rio Preto, RE-Resende, VALValença, VR-Volta Redonda, VAS-Vassouras, TR-Três Rios, P-Petrópolis, RJ-Rio de Janeiro, NI-Niterói. Abbreviations: LN-Liberdade Nappe, ATS-Andrelândia Thrust System, JFTS - Juiz de Fora Thrust System, PSK-Paraiba do Sul Klippe. 


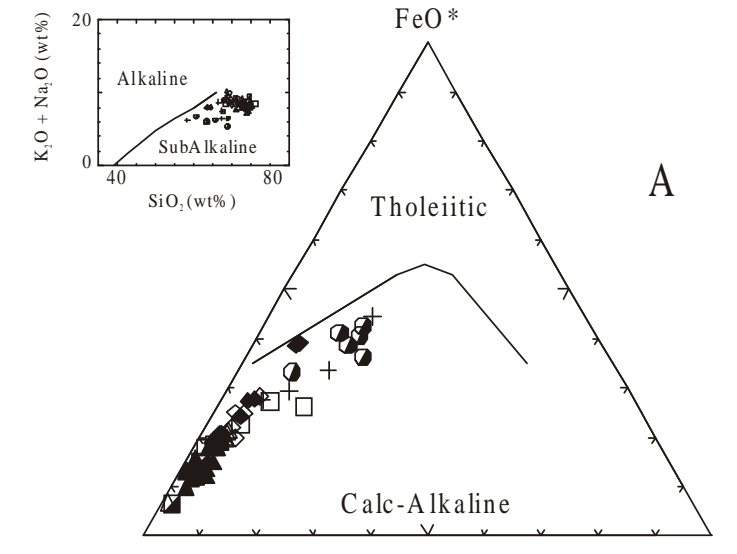

$\mathrm{Na}_{2} \mathrm{O}+\mathrm{K}_{2} \mathrm{O}$

$\mathrm{MgO}$
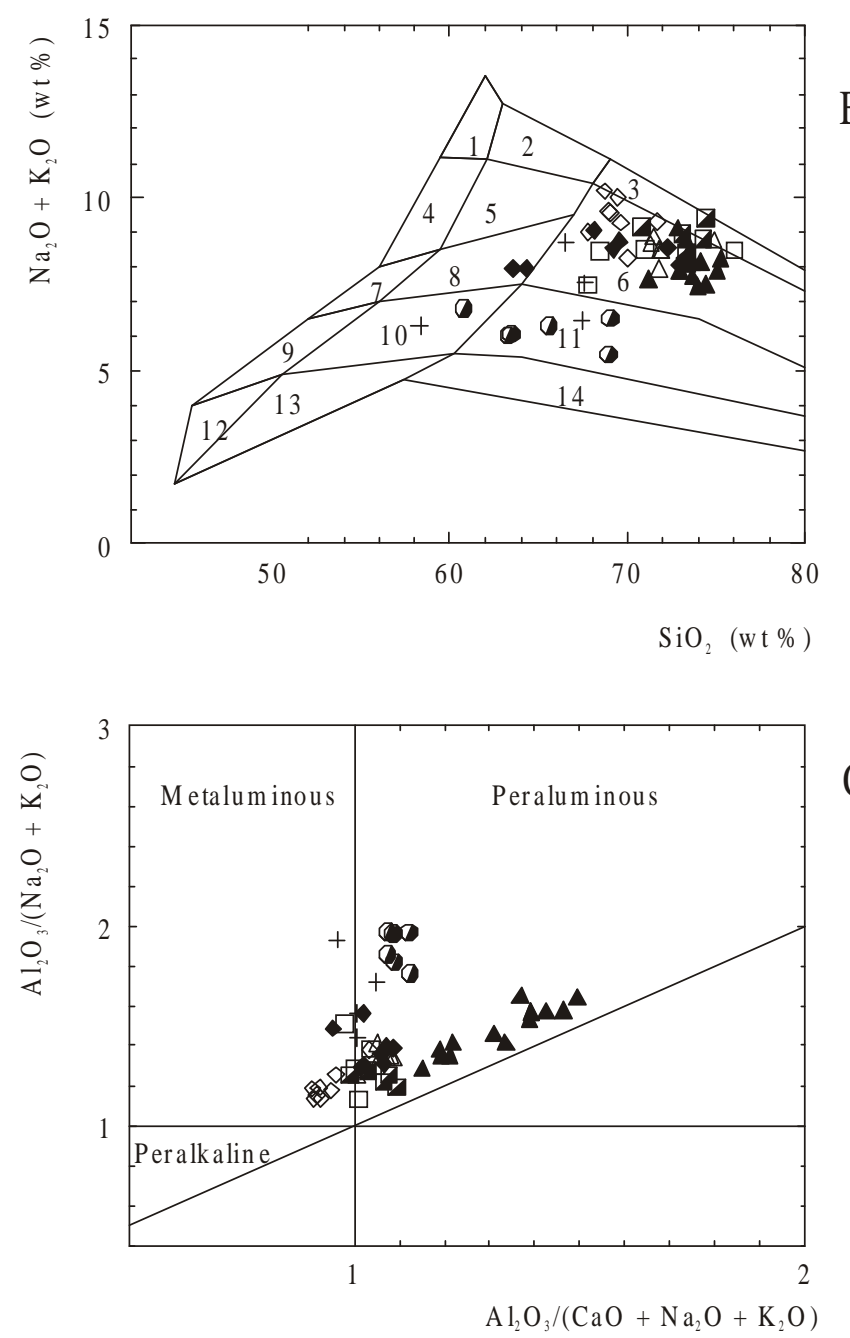

Figure 2 - A-TAS and AFM diagrams. B-Chemical classification (Middlemost 1985); Fields: 3-Alkali-feldspar granite; 6- Granite; 8- Quartz monzonite; 10- Quartz monzodiorite; 11-Granodiorite. C-Shand's index diagram. Symbols: Syn-collisional (syn D1+D2 I-type) Matias Barbosa Granite (cross): Syn-collisional - late D2 I-type plutons: Pedra Selada Granite (closed diamond), Serra do Lagarto Granite (open diamond); Syn-collisional - late D2 S-type plutons: Salvaterra Charnoenderbite (half-closed circle), Capivara Granite (closed triangle); Late-collisional plutons: Getulândia Granite (open square), Fortaleza Granite (half-closed square), Serra do Ipiranga Granite (open triangle).
Charnoenderbite pluton is located on the west side of Juiz de Fora (MG). Fieldwork indicates that its most striking feature is the transition to paragneisses. Oriented schlieren structures, which are parallel to the main regional foliation, resemble ghost features inherited from the preexisting gneisses. An igneous porphyritic texture is locally preserved and phenocrysts include feldspars, garnet and orthopyroxene. Close to the thrust planes and/or sheets, the Salvaterra Charnoenderbite acquires a mylonitic texture resulting from D2 thrusting. Modal analysis and geochemical data (Table 2) reveal a granodioritic composition (Fig. 2B) and a calcalkaline, peraluminous character (Fig. $2 \mathrm{~A}$ and $\mathrm{C}$ ). Among the studied granites, the Salvaterra Charnoenderbite displays the least fractionated REE pattern and an either negative or positive Eu anomaly (Fig. 3B).

The Capivara granite (Almeida 1995) is located near Itamonte (MG) and occurs as a NE-SW lenticular intrusion. Geochemical data (Almeida 1995) indicate a homogeneous granite composition (Fig. 2B) as well as a strongly peraluminous nature (Fig. 2C). This pluton displays a moderate fractionated REE pattern and negative Eu anomaly (Fig. 3B).

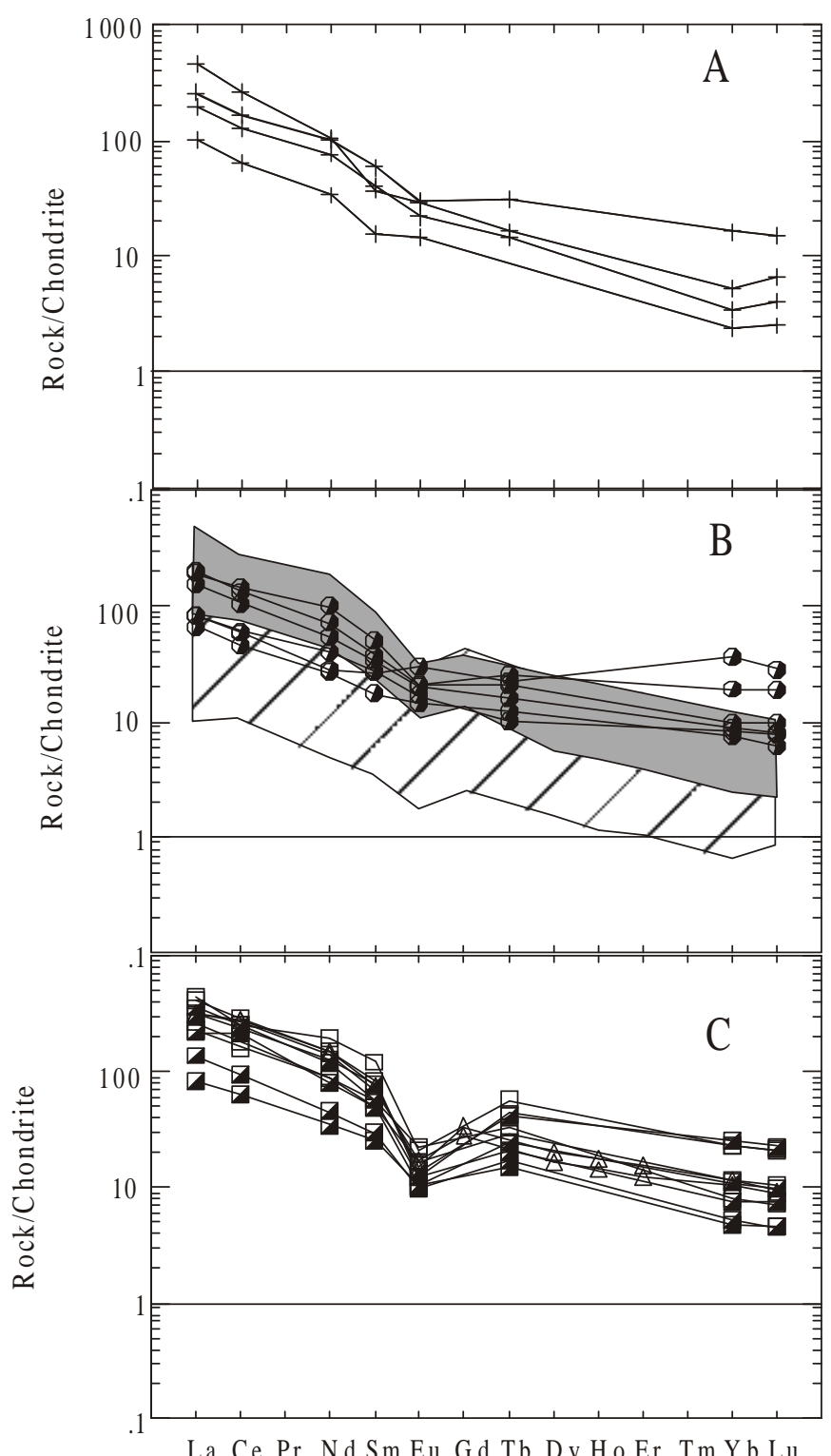

Figure 3 - Chondrite-normalized (Boynton 1984) REE patterns of the studied granites. A - Syn-collisional (syn-D1+D2 I-type) Matias Barbosa Granite (cross); B-Syn-collisional (late-D2 I-type) plutons: Pedra Selada Granite and Serra do Lagarto Granite (gray fill); Syn-collisional (late-D2 S-type) plutons: Salvaterra Charnoenderbite (half-closed circle), Capivara Granite (hatched pattern); C - Late-collisional plutons: Getulândia Granite (open square), Fortaleza Granite (half-closed square), Serra do Ipiranga Granite (open triangle). 
Table 2 - Chemical composition of the syn-collisional (syn D1+D2 pluton) Matias Barbosa Granite (samples 1 to 5) and the syn-collisional (Late-D2) Salvaterra Charnoenderbite (samples 6 to 10).

\begin{tabular}{|c|c|c|c|c|c|c|c|c|c|c|}
\hline $\begin{array}{l}\text { Sampl } \\
\text { e }\end{array}$ & 1 & 2 & 3 & 4 & 5 & 6 & 7 & 8 & 9 & 10 \\
\hline $\mathrm{SiO}_{2}$ & 57.57 & 66.09 & 66.52 & 66.62 & 68.27 & 58.85 & 61.34 & 63.17 & 65.37 & 69.44 \\
\hline $\mathrm{TiO}_{2}$ & 1.14 & 0.67 & 0.69 & 0.71 & 0.52 & 0.99 & 0.72 & 0.66 & 0.73 & 0.52 \\
\hline $\mathrm{Al}_{2} \mathrm{O}_{3}$ & 16.87 & 15.95 & 15.10 & 15.00 & 14.41 & 16.96 & 15.64 & 16.42 & 15.50 & 15.57 \\
\hline $\mathrm{Fe}_{2} \mathrm{O}_{3}{ }^{\mathrm{t}}$ & 8.22 & 4.07 & 4.06 & 4.70 & 5.53 & 6.16 & 6.90 & 6.55 & 6.01 & 4.26 \\
\hline $\mathrm{MnO}$ & 0.15 & - & 0.05 & 0.05 & 0.09 & 0.06 & 0.09 & 0.09 & 0.07 & 0.05 \\
\hline $\mathrm{MgO}$ & 3.02 & 0.97 & 1.37 & 2.01 & 1.70 & 3.14 & 2.55 & 2.58 & 2.34 & 1.12 \\
\hline $\mathrm{CaO}$ & 4.85 & 2.62 & 2.93 & 3.07 & 3.05 & 3.68 & 3.66 & 3.72 & 3.15 & 2.76 \\
\hline $\mathrm{Na}_{2} \mathrm{O}$ & 3.57 & 3.03 & 2.90 & 3.22 & 2.58 & 3.51 & 2.86 & 3.27 & 3.07 & 3.10 \\
\hline $\mathrm{K}_{2} \mathrm{O}$ & 2.65 & 5.64 & 4.53 & 3.17 & 2.84 & 3.08 & 2.98 & 2.76 & 3.20 & 3.46 \\
\hline $\mathrm{P}_{2} \mathrm{O}_{5}$ & 0.68 & 0.30 & 0.28 & 0.21 & 0.09 & 0.46 & 0.18 & 0.34 & 0.24 & 0.32 \\
\hline LOI & 0.95 & 0.60 & 0.45 & 1.20 & 0.60 & 0.63 & 0.74 & 0.59 & 0.76 & 0.29 \\
\hline Total & 99.67 & 99.94 & 98.88 & 99.96 & 99.68 & 97.52 & 97.80 & 100.15 & 100.44 & 100.89 \\
\hline $\mathrm{Cr}$ & - & - & - & - & - & 110 & 88 & 80 & 68 & 36 \\
\hline $\mathrm{Ni}$ & 5 & 2 & 10 & 12 & 21 & 48 & 37 & 38 & 40 & 23 \\
\hline V & 114 & 55 & 53 & 77 & 53 & 79 & 65 & 62 & 71 & 31 \\
\hline $\mathrm{Rb}$ & 165 & 125 & 123 & 114 & 110 & 103 & 115 & 79 & 82 & 82 \\
\hline $\mathrm{Ba}$ & 1787 & 2998 & 986 & 840 & 825 & 857 & 735 & 723 & 845 & 1311 \\
\hline $\mathrm{Sr}$ & 1003 & 854 & 326 & 339 & 272 & 408 & 265 & 284 & 286 & 330 \\
\hline $\mathrm{Hf}$ & 2.60 & 7.80 & 5.10 & 4.40 & 7.90 & 3.50 & 5.30 & 3.20 & 4.20 & 5.50 \\
\hline $\mathrm{Zr}$ & 91 & 338 & 213 & 186 & 267 & 155 & 160 & 471 & 247 & 276 \\
\hline Y & 46 & 13 & 14 & 7 & 44 & 14 & 36 & 26 & 28 & 36 \\
\hline $\mathrm{La}$ & 78.60 & 139.00 & 59.00 & 31.10 & 25.30 & 57.50 & 59.70 & 25.80 & 20.70 & 47.40 \\
\hline $\mathrm{Ce}$ & 133.00 & 211.00 & 102.00 & 51.00 & 47.00 & 113.00 & 105.00 & 49.00 & 37.00 & 86.00 \\
\hline $\mathrm{Nd}$ & 60.00 & 63.00 & 45.00 & 20.00 & 17.00 & 58.00 & 42.00 & 24.00 & 16.00 & 33.00 \\
\hline $\mathrm{Sm}$ & 11.20 & 7.23 & 7.80 & 3.02 & 5.16 & 9.40 & 7.10 & 5.30 & 3.40 & 6.60 \\
\hline $\mathrm{Eu}$ & 2.17 & 2.10 & 1.60 & 1.07 & 2.20 & 1.50 & 1.50 & 1.24 & 1.07 & 1.50 \\
\hline $\mathrm{Tb}$ & 1.50 & 0.80 & 0.70 & - & 1.10 & 1.00 & 1.20 & 0.50 & 0.60 & 0.80 \\
\hline $\mathrm{Yb}$ & 3.40 & 1.10 & 0.70 & 0.50 & 7.50 & 2.00 & 3.90 & 1.75 & 1.57 & 1.85 \\
\hline $\mathrm{Lu}$ & 0.48 & 0.21 & 0.13 & 0.08 & 0.91 & 0.31 & 0.59 & 0.25 & 0.20 & 0.26 \\
\hline
\end{tabular}

Analyses carried out at ACTLABS (Canadá): major and trace elements - ICP fusion; REE - INAA. XRF and ICP analyses for the Serra do Ipiranga Granite were carried out at GEOSOL Laboratory (Brazil). Major and trace elements are expressed in \% wt and ppm, respectively.

Table 3 - Chemical compositions of the late-collisional granites from the Paraíba do Sul Klippe. Getulândia Granite (samples 11 to 14), Fortaleza Granite (samples 15 to 19) and Serra do Ipiranga Granite (samples 20 to 25).

\begin{tabular}{|c|c|c|c|c|c|c|c|c|c|c|c|c|c|c|c|}
\hline $\begin{array}{l}\text { Sampl } \\
\text { e } \\
\end{array}$ & 11 & 12 & 13 & 14 & 15 & 16 & 17 & 18 & 19 & 20 & 21 & 22 & 23 & 24 & 25 \\
\hline $\mathrm{SiO}_{2}$ & 66.19 & 67.45 & 69.62 & 74.63 & 70.13 & 70.38 & 71.34 & 73.05 & 73.48 & 70.50 & 70.80 & 70.80 & 71.20 & 71.20 & 74.30 \\
\hline $\mathrm{TiO}_{2}$ & 0.74 & 0.55 & 0.39 & 0.18 & 0.30 & 0.17 & 0.09 & 0.24 & 0.23 & 0.31 & 0.32 & 0.33 & 0.33 & 0.34 & 0.06 \\
\hline $\mathrm{Al}_{2} \mathrm{O}_{3}$ & 14.36 & 15.19 & 14.16 & 11.98 & 14.48 & 14.18 & 13.79 & 13.83 & 13.44 & 14.60 & 14.60 & 14.40 & 14.40 & 14.80 & 13.80 \\
\hline $\mathrm{Fe}_{2} \mathrm{O}_{3}$ & 3.85 & 3.69 & 2.93 & 2.07 & 2.51 & 1.35 & 0.70 & 2.20 & 1.75 & 0.70 & 0.83 & 0.82 & 0.59 & 0.74 & 0.17 \\
\hline $\mathrm{FeO}$ & & & & & & & & & & 1.80 & 1.50 & 1.50 & 1.90 & 1.40 & 0.49 \\
\hline $\mathrm{MnO}$ & 0.06 & 0.06 & 0.07 & 0.03 & 0.04 & 0.03 & 0.03 & 0.03 & 0.06 & 0.03 & 0.03 & 0.03 & 0.03 & 0.03 & 0.01 \\
\hline $\mathrm{MgO}$ & 1.12 & 1.93 & 0.69 & 0.20 & 0.50 & 0.35 & 0.19 & 0.37 & 0.29 & 0.49 & 0.41 & 0.51 & 0.49 & 0.50 & 0.11 \\
\hline $\mathrm{CaO}$ & 1.93 & 3.03 & 1.74 & 0.71 & 1.68 & 1.03 & 0.59 & 1.43 & 0.88 & 1.50 & 1.50 & 1.50 & 1.40 & 2.00 & 1.50 \\
\hline $\mathrm{Na}_{2} \mathrm{O}$ & 2.66 & 3.50 & 3.49 & 2.81 & 3.10 & 3.49 & 3.12 & 3.11 & 2.87 & 2.80 & 3.00 & 2.70 & 2.90 & 3.40 & 2.80 \\
\hline $\mathrm{K}_{2} \mathrm{O}$ & 5.52 & 3.96 & 4.88 & 5.52 & 5.98 & 5.14 & 5.92 & 5.28 & 5.84 & 5.80 & 5.80 & 5.70 & 5.50 & 4.50 & 5.90 \\
\hline $\mathrm{P}_{2} \mathrm{O} 5$ & 0.32 & 0.20 & 0.18 & 0.06 & 0.34 & 0.16 & 0.10 & 0.10 & 0.08 & 0.10 & 0.11 & 0.11 & 0.10 & 0.11 & 0.05 \\
\hline $\mathrm{PF}$ & 1.05 & 1.20 & 1.53 & 0.89 & 0.98 & 1.42 & 2.06 & 1.17 & 0.87 & 0.85 & 0.62 & 1.17 & 0.68 & 0.63 & 0.72 \\
\hline Total & 97.80 & 100.76 & 99.68 & 99.08 & 100.04 & 97.70 & 97.93 & 100.81 & 99.79 & 99.48 & 99.52 & 99.57 & 99.52 & 99.65 & 99.89 \\
\hline $\mathrm{Cr}$ & 11 & 55 & 2 & 1 & 9 & 7 & 4 & 2 & 4 & & & & & & \\
\hline $\mathrm{Ni}$ & 5 & 20 & 2 & 1 & 3 & 2 & 1 & 2 & 2 & & & & & & \\
\hline $\mathrm{Rb}$ & 284 & 206 & 168 & 244 & 343 & 301 & 234 & 297 & 409 & 310 & 300 & 310 & 290 & 250 & 260 \\
\hline $\mathrm{Ba}$ & 737 & 861 & 729 & 468 & 1184 & 529 & 796 & 1248 & 382 & 770 & 710 & 730 & 760 & 680 & 680 \\
\hline $\mathrm{Sr}$ & 153 & 305 & 365 & 59 & 193 & 139 & 263 & 199 & 87 & 170 & 170 & 170 & 170 & 210 & 190 \\
\hline $\mathrm{Zr}$ & 328 & 191 & 267 & 248 & 164 & 114 & 76 & 213 & 205 & 330 & 360 & 380 & 330 & 390 & 60 \\
\hline $\mathrm{La}$ & 135.4 & 70.91 & 82.02 & 126.29 & 96.31 & 42.27 & 25.66 & 111.4 & 67.23 & & 98.17 & & & 97.51 & \\
\hline $\mathrm{Ce}$ & 203.62 & 130.57 & 147.73 & 234.24 & 191.8 & 75.82 & 51.11 & 204.74 & 174.89 & & 224.70 & & & 220.40 & \\
\hline $\mathrm{Nd}$ & 117.83 & 54.24 & 52.98 & 89.62 & 75.71 & 27 & 20.86 & 71.26 & 48.52 & & 88.96 & & & 85.00 & \\
\hline $\mathrm{Sm}$ & 23.77 & 11.05 & 9.58 & 16.29 & 15.14 & 5.71 & 4.9 & 11.04 & 9.6 & & 14.50 & & & 13.02 & \\
\hline $\mathrm{Eu}$ & 1.56 & 1.64 & 1.21 & 0.9 & 1.02 & 0.72 & 0.78 & 0.9 & 0.76 & & 1.08 & & & 1.24 & \\
\hline Gd & & & & & & & & & & & 8.61 & & & 7.27 & \\
\hline $\mathrm{Tb}$ & 2.79 & 1.61 & 1.43 & 2.14 & 2.02 & 0.83 & 0.73 & 1 & 1.21 & & & & & & \\
\hline Dy & & & & & & & & & & & 6.48 & & & 5.27 & \\
\hline Ho & & & & & & & & & & & 1.26 & & & 1.02 & \\
\hline Er & & & & & & & & & & & 3.19 & & & 2.60 & \\
\hline $\mathrm{Yb}$ & 4.83 & 1.69 & 2.38 & 4.77 & 5.35 & 1.09 & 0.99 & 1.6 & 2.29 & & 2.37 & & & 2.16 & \\
\hline $\mathrm{Lu}$ & 0.67 & 0.23 & 0.34 & 0.68 & 0.73 & 0.15 & 0.15 & 0.24 & 0.31 & & 0.30 & & & 0.29 & \\
\hline
\end{tabular}

Analyses were carried out at ACTLABS (Canadá): major and trace elements - ICP fusion; REE - INAA. XRF and ICP analyses for the Serra do Ipiranga Granite were carried out at GEOSOL

Laboratory (Brazil). Major and trace elements are expressed in \%wt and ppm, respectively. 
Late-collisional magmatism This widespread magmatism within the Paraíba do Sul Klippe can be characterized by subvertical dykes and NE-SW elongated plutons related to the late deformation event structures (D3 shear zones). Expressive examples include the Serra do Ipiranga pluton (Heilbron et al. 1992), nearby Barra do Piraí, and the Getulândia and Fortaleza granites (Valladares et al. 1995, Valladares 1996) between Barra Mansa and Rio Claro. These intrusions are homogeneous in composition, being granites s.s. (Fig. 2B), and bear biotite as the mafic mineral. These plutons have a NESW weak foliation interpreted as magmatic flow foliation in the Serra do Ipiranga (Heilbron and Machado 1995) and Fortaleza intrusions. Geochemical data (Table 3) are typical of I-type granites with a slightly peraluminous character (Shand's index between 1,0 and 1,1) Chondrite-normalized REE patterns are fractionated and display a pronounced negative Eu anomaly (Fig. 3C). The most reliable geochronological data representing this magmatic event are the two U$\mathrm{Pb}$ monazite analyses of presented by Machado et al. (1996) and Valladares (1996) for the Getulândia Granite. These data yield concordant ages of $527 \pm 3 \mathrm{Ma}$ and $535 \pm 3 \mathrm{Ma}$ and are taken as the minimum age for movement in the shear zone along which the Getulândia pluton was emplaced during stage D3. The only available $\mathrm{Nd}$ isotopic data yield a Transamazonian depleted mantle model age $\left(\mathrm{T}_{\mathrm{DM}}\right.$, Table 1), suggesting that it might have derived from reworked basement rocks.
CONCLUDING REMARKS Granite magmatism in the study area can be divided into three stages based on U-Pb geochronology, geochemistry and structural geology (Table 4): 1) the syn-collisional stage 1 (595-565 Ma) is represented by foliated, peraluminous S-type and metaluminous I-type granites; 2 ) the syn-collisional stage 2 (565$540 \mathrm{Ma}$ ) comprises weakly foliated metaluminous I-type granites with basic enclaves, peraluminuous S-type granites and minor leucogranites; 3 ) the late-collisional stage (540-520 Ma) includes alkali-calcic, slightly peraluminous leucogranites which occur as subvertical dykes and late shear zones-related plutons. Preliminary $\mathrm{Sm}-\mathrm{Nd}$ isotopic data indicate that the late-collisional granites might have derived from reworked basement rocks. The syn-collisional $1 \mathrm{~S}-$ type granites were generated by melting of mixed sources, possibly Paleoproterozoic metasediments and 1,0-0,9 Ga basic rocks, or yet another unknown Mesoproterozoic source.

Table 4 summarizes the relationships between magmatism and tectonics within the central segment of the Ribeira fold belt, including a comparison between the magmatic record on the Occidental and Oriental terranes. The time span proposed for the pre-collisional stage (restricted to the Oriental terrane) is based on U-Pb geochronological data in Tupinambá (1999).

Acknowledgements To FAPERJ (Research Foundation of State of Rio de Janeiro) for the financial support, to S. Valente for the English review and to two anonymous RBG referees for the critical review of the manuscript.

Table 4 - General characteristics of the Brasiliano granites of the Occidental and Oriental terranes, according to the stage of orogeny and time relationships with deformation phases.

\begin{tabular}{|c|c|c|c|c|c|c|c|}
\hline $\begin{array}{c}\text { Stage of } \\
\text { orogeny / } \\
\text { deformation }\end{array}$ & $\begin{array}{l}\text { Time } \\
\text { span } \\
(\mathrm{Ma})\end{array}$ & Geochemistry & Plutons/Age (Ma) & Structures & $\begin{array}{l}\text { Tectonic } \\
\text { Domains }\end{array}$ & $\begin{array}{l}\text { Tectonic } \\
\text { setting }\end{array}$ & $\begin{array}{r}\text { Selected } \\
\text { reference } \\
\mathrm{s}\end{array}$ \\
\hline $\begin{array}{l}\text { Slab } \\
\text { detachment - } \\
\text { related } \\
\text { granites }\end{array}$ & $<520$ & $\begin{array}{l}\text { - Calc-alkaline to alkali- } \\
\text { calcic plutons } \\
\text { - Tholeiitic gabbros and } \\
\text { diorites }\end{array}$ & & $\begin{array}{l}\text { Non-foliated and mostly } \\
\text { zoned stocks and sheets. } \\
\text { Primary flow foliation and } \\
\text { layering. Dykes. Possible } \\
\text { mingling and mixing } \\
\text { processes }\end{array}$ & $\begin{array}{l}\text { Recorded } \\
\text { in both } \\
\text { tectonic } \\
\text { terranes }\end{array}$ & $\begin{array}{l}\text { Extensional } \\
\text { collapse of } \\
\text { the orogenic } \\
\text { belt }\end{array}$ & $\begin{array}{l}\text { (1) } \\
(2) \\
(3) \\
(4),(5), \\
(12) \\
(18) \\
\end{array}$ \\
\hline $\begin{array}{l}\text { Late- } \\
\text { collisional } \\
\text { (syn-D3) }\end{array}$ & $540-520$ & $\begin{array}{l}\text { - Alkali-calcic stocks of } \\
\text { slightly peraluminous } \\
\text { leucogranites }\end{array}$ & $\begin{array}{l}\text { - Getulândia (535- } \\
\text { 528Ma) Fortaleza and } \\
\text { Serra do Ipiranga. }\end{array}$ & $\begin{array}{l}\text { D3 shear zones-related } \\
\text { subvertical dykes and } \\
\text { weak foliation along } \\
\text { contacts }\end{array}$ & $\begin{array}{l}\text { Recorded } \\
\text { in both } \\
\text { tectonic } \\
\text { terranes }\end{array}$ & $\begin{array}{l}\text { Crustal } \\
\text { reworking of } \\
\text { basement and } \\
\text { cover rocks }\end{array}$ & $\begin{array}{l}(5),(6), \\
(7),(8), \\
(9),(10), \\
(11), \\
(12),(19)\end{array}$ \\
\hline $\begin{array}{l}\text { Syn- } \\
\text { collisional } 2 \\
\text { (Late-D2) }\end{array}$ & $565-540$ & $\begin{array}{l}\text { - Abundant metaluminous } \\
\text { I-type granites with basic } \\
\text { enclaves } \\
\text { - Peraluminous S-type } \\
\text { granites } \\
\text { - Subordinated two-mica } \\
\text { peraluminous S-type } \\
\text { leucogranites }\end{array}$ & $\begin{array}{l}-\quad \text { Serra do Lagarto; } \\
\text { Pedra Selada and } \\
\text { Taquaral (553Ma) } \\
\text { - Salvaterra } \\
\text { Charnoenderbite } \\
\text { - } \quad \text { Capivara }\end{array}$ & $\begin{array}{l}\text { Weakly foliated plutons } \\
\text { and sheets }\end{array}$ & $\begin{array}{l}\text { Recorded } \\
\text { in both } \\
\text { tectonic } \\
\text { terranes }\end{array}$ & $\begin{array}{l}\text { Crustal } \\
\text { reworking of } \\
\text { basement and } \\
\text { cover rocks }\end{array}$ & $\begin{array}{l}(8),(9) \\
(10),(13) \\
(14) \\
(15) \\
(17) \\
(20)\end{array}$ \\
\hline $\begin{array}{l}\text { Syn- } \\
\text { collisional } 1 \\
\text { (Syn- } \\
\text { D1+D2) } \\
\end{array}$ & $595-565$ & $\begin{array}{l}\cdot \quad \text { Abundant peraluminous } \\
\text { S-type granites } \\
-\quad \text { Metaluminous I-type } \\
\text { granites }\end{array}$ & $\begin{array}{l}\text { - } \quad \text { Rio Turvo } \\
\text { batholith (579Ma) } \\
\text { - Matias Barbosa }\end{array}$ & $\begin{array}{l}\text { Foliated and mylonitic } \\
\text { plutons }\end{array}$ & $\begin{array}{l}\text { Recorded } \\
\text { in both } \\
\text { tectonic } \\
\text { terranes }\end{array}$ & $\begin{array}{l}\text { Crustal } \\
\text { reworking of } \\
\text { basement and } \\
\text { cover rocks }\end{array}$ & $\begin{array}{l}(5) \\
(8) \\
(9) \\
(10),(17)\end{array}$ \\
\hline $\begin{array}{l}\text { Pre- } \\
\text { collisional } \\
\text { (Pre-D1) }\end{array}$ & $630-595$ & $\begin{array}{l}\text { - Tonalites to } \\
\text { granodiorites and tholeiitic } \\
\text { gabbros }\end{array}$ & & $\begin{array}{l}\text { Foliated plutons. Mylonitic } \\
\text { structures associated with } \\
\text { tectonic boundaries }\end{array}$ & $\begin{array}{l}\text { Only } \\
\text { within } \\
\text { Oriental } \\
\text { terrane } \\
\end{array}$ & $\begin{array}{l}\text { Cordilleran } \\
\text { Magmatic } \\
\text { Arc }\end{array}$ & $\begin{array}{l}(4) \\
(16)\end{array}$ \\
\hline
\end{tabular}

Selected references: (1) Pires et al., 1982; (2) Wiedemann, 1993; (3) Junho, 1993; (4) Figueiredo \& Campos Neto, 1993; (5) Machado, 1997; (6) Machado \& Demange, 1994, (7, 8) Heilbron, 1993, 1995, (9) Heilbron et al., 1995; (10) Machado et al., 1996, (11) Valladares, 1996; (12) Porto Jr. 1994; (13) Grossi Sad \& Barbosa, 1985; (14) Tupinambá, 1993; (15) Junho et al., 1999; (16) Tupinambá et al., 1998; (17) Duarte, 1998; (18) Nogueira, 1993; (19) Valladares et al. 1995; (20) Duarte et al. ,1999. 


\section{References}

Almeida M.E. 1995. Geologia, petrografia e geoquímica preliminar do leucogranito Capivara, Itamonte ( $M G$ ). Inst. de Geociências, Universidade Federal do Rio de Janeiro, Rio de Janeiro, Dissertação de Mestrado, 132p.

Boynton W.R. 1984. Cosmochemistry of the rare earth elements meteorite studies. In P. Henderson (ed) Rare Earth Element Geochemistry. Amsterdan. 63 -114

Duarte P.D. 1998. Evolução tectônica dos ortognaisses dos Complexos Juiz de Fora e Mantiqueira na região de Juiz de Fora, M.G.: Geologia, petrologia e geoquímica. Instituto de Geociências, Universidade de São Paulo, São Paulo, Tese de Instituto de Geociên

Duarte B.P., Heilbron M., Campos Neto M. C., Porto Jr. R. 1999. The Garnet Charnockite Plutonic Body of the Juiz de Fora Region, Central Segment of the Pan-AfricanBrasiliano Ribeira Belt, Southeastern Brazil. In : SBG, Simp. Nac. Est. Tect., 7 , Lençóis, Resumos Expandidos, 2:56-57.

Figueiredo M.C.H. and Campos Neto M.C. 1993. Geochemistry of the Rio Doce Magmatic Arc, Southeastern Brazil. Anais Acad. Bras. Ci, 65 (supl. 1):63-81.

Grossi Sad J.H. and Barbosa A.L.M. 1985. A origem dos charnoquitos e rochas afins da região do médio Paraíba do Sul, Estado do Rio de Janeiro. In: SBG, Belo Horizonte, Boletim Especial, 15-27.

Heilbron M. 1993. Evolução tectono-metamórfica da seção Bom Jardim de Minas-MGBarra do Piraí-RJ, setor central da Faixa Ribeira. Instituto de Geociências, Universidade de São Paulo, São Paulo, Tese de Doutoramento, 268p.

Heilbron M. 1995. O Segmento Central da Faixa Ribeira: Síntese Geológica e Ensaio de Evolução Geotectônica. Instituto de Geociências, Universidade do Estado do Rio de Janeiro, Rio de Janeiro, Tese de Livre Docência, 140 p.

Heilbron M. and Machado R. 1995. O magmatismo tardi- a pós-colisional Brasiliano na seção entre Bom Jardim de Minas e Barra do Piraí, Segmento Central da Faixa Ribeira. In: SBG, Simp. Geol. Sudeste, 4, Águas de São Pedro, Boletim de Resumos, 140

Heilbron M., Mohriak W., Valeriano C.M., Milani E., Almeida J.C.H, Tupinambá M. 1999 In: W. U. Morhriak and M. Talwani (eds). From Collision to Extension: The Root of the Southeastern Continental Margin of Brazil.Geology and Geophysics of Continental Margins. AGU Geophysical Monograph, 115:1-31.

Heilbron M., Santos R.O., Valladares C.S., Valeriano C.M. 1992 Geologia e litogeoquímica do Leucogranito Serra do Ipiranga. In: SBG, Cong. Bras. Geol., 37, São Paulo. Boletim de Resumos Expandidos, 375-376.

Heilbron M., Tupinambá M., Almeida J.C.H., Valeriano C.M., Valladares C.S., Duarte B.P. 1998. New constraints on the tectonic organization and structural styles related to the Brasiliano collage of the central segment of the Ribeira belt, SE Brazil. In: SBG, International Conference on Basement Tectonics, 14, Ouro Preto, Extended International Con

Junho M.C.B. 1993. Granitóides Brasilianos na região central do Estado do Rio de Janeiro- Geoquímica preliminar. Anais Acad. Bras. Ci., 65:161-179.

Junho M.C.B. 1995. Leucogranites and related migmatites, southern Minas Gerias and southwestern Rio de Janeiro states, Brazil. Anais Acad. Bras.Ci., 67):498-511.

Junho M.C.B., Heilbron M., Valeriano C.M. 1999. Porphyritic I-type syntectonic granite and related rocks, Ribeira mobile belt, souwestern Rio de Janeiro state, Brazil. Anais Acad. Bras.Ci., 71:631-647.
Machado N, Valladares C.S., Heilbron M., Valeriano C.M. 1996. U-Pb geocronology of the central Ribeira Belt (Brazil) and implications for the evolution of the Brazilian Orogeny. Prec. Research, 79:347-367.

Machado R. 1997. Litogeoquímica e tectônica dos granitóides Neoproterozóicos do Cinturão Paraíba do Sul no Estado do Rio de Janeiro. Instituto de Geociências, Universidade de São Paulo, São Paulo, Tese de Livre Docência , 215p.

Machado R. and Démange M. 1991. Contexto Tectônico e estrutural dos granitóides brasilianos do estado do Rio de Janeiro. In: SBG, Simp. Nac. Est. Tect., 3, Rio Claro, Boletim de Resumos, 64-65.

Machado R. and Démange M. 1994. Classificação estrutural e tectônica dos granitóides Neoproterozóicos do Cinturão Paraíba do Sul no Estado do Rio de Janeiro. Boletim do IG-USP, 25:81-96

Middlemost E.A.K. 1985. Magmas and magmatic rocks. ed. London, Longman press, 266p.

Nogueira J.R. 1994. Relações tectonoestruturais e metamórficas entre metassedimentos e ortognaisses em facies granulito na região à sudoeste de Juiz de Fora, MG. Instituto de Geociências, Universidade do Estado do Rio de Janeiro, Rio de Janeiro, Dissertação de Mestrado, 151p.

Pires F.M., Valença J.G., Ribeiro A 1982. Multistage generation of granite in Rio de Janeiro, Brazil. . Anais Acad. Bras. Ci., 54:563-574.

Porto Jr. R. 1994. Petrologia das Rochas Graníticas das Serras da Pedra Branca e Misericórdia, Município do Rio de Janeiro, RJ, Brasil. Instituto de Geociências, Universidade do Estado do Rio de Janeiro, Rio de Janeiro, Dissertação de Mestrado, 222p.

Tupinambá M. 1993. Rochas intrusivas e metassedimentos granulíticos do Complexo Paraíba do Sul na parte setentrional da Faixa Ribeira. In: SBG, Simp. Geol. Sud., Rio de Janeiro, Atas: 187-193

Tupinambá M. 1999. Evolução tectônica e magmática da Faixa Ribeira na região serrana do Estado do Rio de Janeiro. Instituto de Geociências, Universidade de São Paulo, São Paulo, Tese de Doutoramento, 221p.

Valladares C.S., Heilbron M., Figueiredo M.C.H. 1995. O Granito Getulândia e sua relação com a Zona de Cisalhamento Taxaquara. In : SBG, Simp. Nac. Est. Tect., 5, Gramado, Anais: 217-218.

Valladares C. S. 1996. Evolução geológica do Complexo Paraíba do Sul, no segmento central da Faixa Ribeira, com base em estudos de geoquímica e de geocronologia $U-P b$. Instituto de Geociências, Universidade de São Paulo, São Paulo, Tese de Doutoramento, $147 \mathrm{p}$

Wiedemann C. M. 1993. Early Paleozoic, late- to post-collisional magmatic arc of the coastal mobile belt in the state of Espírito Santo, eastern Brazil, Anais Acad. Bras. Ci., 65(supl 1):162-181.
Contribution IGC-009 Received January 22, 2000
publication May 16, 2000 